\title{
Metabolic disturbances identified by SPECT-CT in patients with a clinical diagnosis of sacroiliac joint incompetence
}

\author{
Mel Cusi · Jennifer Saunders · Hans Van der Wall • \\ Ignac Fogelman
}

Received: 23 August 2012/Revised: 30 January 2013/Accepted: 18 February 2013/Published online: 1 March 2013

(C) Springer-Verlag Berlin Heidelberg 2013

\begin{abstract}
Purpose To establish the sensitivity and specificity of cross-sectional scintigraphy [single photon emission computed tomography (SPECT)] combined with computed $\mathrm{X}$-ray tomography $(\mathrm{CT})$ in the detection of sacroiliac joint (SIJ) mechanical dysfunction and evaluate reproducibility of reporting.

Methods Patients with pelvic girdle pain either on the basis of peri-partum SIJ dysfunction or trauma were included. These patients were imaged with bone scintigraphy with hybrid imaging with SPECT/CT.

Results The study group comprised 100 patients (72 females, 28 males). Trauma accounted for $52 \%$ and the remainder were patients with peri-partum pain. Average age was 43 years and average length of history was $>2$ years. The major finding was increased uptake in the upper SIJ and posterior soft-tissues/ligaments. Hybrid imaging had a sensitivity of $95 \%$ and specificity of $99 \%$. Positive predictive value was $99 \%$ and negative predictive value $94 \%$. Power of the test was 1.0. Reproducibility of the test was good with kappa values of 0.85 .
\end{abstract}

M. Cusi

Sydney School of Medicine, University of Notre Dame,

Fremantle, Australia

J. Saunders

University of Sydney, Sydney, Australia

H. Van der Wall $(\bowtie)$

Concord Nuclear Imaging, 4 Hospital Road,

Concord West, Sydney, NSW 2138, Australia

e-mail: hvanderwall@gmail.com

I. Fogelman

Departments of Nuclear Medicine,

Guys and St. Thomas' Hospital, London, UK
Conclusion Hybrid imaging with SPECT/CT reproducibly demonstrates metabolic alterations around the SIJ in patients with SIJ dysfunction, which we have termed SIJ incompetence. The condition is more common than previously recognised and frequently occurs after trauma, which has not been reported previously.

Keywords Sacroiliac joint $\cdot$ SPECT/CT $\cdot$ Sacroiliac joint incompetence $\cdot$ Pain provocation tests $\cdot$ Pain palpation tests

\section{Introduction}

The sacroiliac joint (SIJ) as a cause of clinical symptoms is a poorly recognised and controversial subject. In the last decade, it has been increasingly recognised as a major source of lower back pain in the peri-partum period, predominantly in the Northern European literature. The SIJ was raised as a source of lower back pain as early as 1905 by Goldthwaite [1]. Unfortunately, the subject was lost in the noise of the general medical literature following the publication of the classic 1934 article on lower back pain by Mixter and Barr [2]. In this article, the intervertebral disc was ascribed as a common cause of lower back and leg pain.

The SIJ may be the source of pain in the lower back and buttocks in up to $15-21 \%$ of the population [3]. Pelvic girdle pain (PGP) is a specific form of low back pain that may occur separately or in conjunction with low back pain in pregnancy or the post-partum period [4]. There are three prospective studies [5-7] that report a relatively high incidence and point prevalence of PGP in pregnancy in large cohorts totalling almost 2,000 patients. The results indicate that approximately $20 \%$ of pregnant women suffer from PGP. Furthermore, there is growing evidence that 
points to the SIJ as an important source of PGP. The prevalence of SIJ pain would appear to be at least $13 \%$ and perhaps as high as $30 \%[8,9]$.

The diagnosis of PGP can be reached after exclusion of the lumbar spine as a cause of symptoms. Imaging has a role in exclusion of significant pathology in the lumbar spine, but has been unable to detect mechanical disturbances in the SIJ. The failure to detect mechanical pathology in the SIJ, apart from fractures and inflammatory disease has been shown for CT [10, 11], MRI [12, 13], scintigraphy using tomography [14], and ultrasound [15]. As a result, the diagnosis of SIJ dysfunction must be based on history and physical examination and this has been established with high levels of evidence and reproducibility in the literature [3, 12, 16-23]. Patterns of pain and/or functional disturbances relating to PGP must be reproducible by specific clinical tests [4, 19]. PGP was initially defined in patients who were pregnant or in the post-partum period [5-7]. We have found a population of patients with a similar clinical presentation, but with a variety of traumatic aetiologies in both males and females. We have termed this condition SIJ incompetence as the population assessed included female and male patients with a history of trauma, thus falling outside the established definition and causation of PGP [5-7]. Both PGP and SIJ incompetence are frequently misdiagnosed as non-specific low back pain because there is poor clinical recognition of the condition and imaging tests such as MRI fail to detect the changes around the SIJ [12, 13]. Firm diagnostic clinical criteria for PGP have been established in the last few years [4]. A prospective study was therefore undertaken to evaluate the clinical findings of SIJ incompetence in patients with a high pre-test probability based on history and physical examination. Those patients with a high test probability were then imaged with bone scintigraphy [single photon emission computed tomography (SPECT)] in combination with X-ray computed tomography (CT) to examine the possibility of detecting metabolic disturbances in and around the pelvic girdle. Based on a number of early observations, we hypothesised that scintigraphy could detect several subtle changes in and around the SIJ and tendon attachments on the pelvis, which were specific to the condition. Control groups were also examined and imaged to assess the specificity of clinical and potential imaging findings. The control groups included both patients with no symptoms and a group with non-specific low back pain.

\section{Materials and methods}

\section{Population}

The study population of 100 consecutive patients was comprised of 72 females and 28 males with an average age of 43 years (range: 21-78 years, median age: 40 years). All patients fulfilled the clinical criteria for a diagnosis of SIJ incompetence (at least 3/4 positive clinical tests) [4]. The main clinical symptom was of low back or buttock pain of at least 3 months duration. The control group of 80 cases was scanned for staging of prostate carcinoma (ten cases with no hip or low back pain), suspected hip pathology $(n=20)$ or non-specific low back pain $(n=50)$. There were 44 females and 36 males. The average age was 45 years (range: $16-71$ years, median age: 49 years).

\section{Ethics approval}

The prospective part of the study was approved as part of a prolotherapy trial incorporating bone scintigraphy before and after prolotherapy for PGP by the Institutional Ethics Committee of the Sydney Medical School, University of Notre Dame, Australia.

\section{Clinical assessment}

The 100 index patients were clinically assessed by two experienced sport medicine physicians (MC and JS) with a battery of tests [3, 19, 21, 23-28]. A failure of load transfer across the SIJ was defined as having three or more of four tests positive [4]. Briefly, the evidence-based diagnosis of PGP/SIJ incompetence has been defined under the European Commission, Research Directorate-General, Department of Policy, Coordination and Strategy (Working Group 4) [4]. A diagnosis may be made by pain provocation tests (posterior pelvic pain provocation test/thigh thrust, Patrick's Faber) and pain palpation tests (long dorsal ligament test and palpation of the symphysis). As a functional test, the active straight leg raise (ASLR) test is also a valid inclusion [4]. The Stork test (stance phase) is a good indicator of muscle recruitment patterns [29].

The control group of 80 patients was examined to ensure that none of the tests were positive prior to inclusion in the study.

\section{SPECT/CT imaging}

Patients were injected with $\sim 1.0 \mathrm{GBq}$ of $99 \mathrm{~m} \mathrm{Tc} \mathrm{HDP}$ and a dynamic and blood pool image of the anterior and posterior pelvis obtained on the Hawkeye 4 Hybrid CT/ Gamma camera (General Electric, Milwaukee, WI, USA) immediately (Dynamic) and 3-5 min after injection (blood pool). Delayed planar images of the anterior and posterior pelvis were obtained at $2.5-3.0 \mathrm{~h}$ after injection. SPECT images were acquired at 64 stops of $20 \mathrm{~s}$ per stop in a circular orbit. CT images were then acquired over $360^{\circ}$ at a low dose of 45-50 mAS. CT images were acquired from 
the level of the lesser trochanters to approximately the L3 level. The SPECT image sets were iteratively reconstructed by ordered subset expectation maximisation (OSEM), filtered, attenuation corrected and fused with the CT images using the Xeleris display software (General Electric). Semi-quantitative analysis was performed by obtaining counts from a standard region of interest over the softtissues posterior to the SIJ at the level of the S2 segment. Sampling was obtained from the identical site on both sides using the CT study as a guide. Uptake was assessed relative to the contralateral structure or the adjacent ilium if the condition was bilateral.

The specific information obtained from each SPECT/CT study was as follows.

1. SIJ uptake, posterior soft-tissue uptake, ligamentous uptake at insertion into the ilium (loss of normal "dumbbell" configuration).

2. CT grading of sclerosis/erosive disease in the upper SIJ as normal, mild, medium or marked.

3. Uptake around the pubic symphysis or at the adductor/ hamstring tendon insertions and the CT appearances of these sites.

4. Evidence of lumbar zygapophyseal joint uptake or uptake around the disc spaces with comment on CT appearance of the joints and intervertebral discs.

5. Comments on any abnormal uptake and CT appearances of the hips.

\section{Statistical analysis}

The population demographics of the index group with PGP/SIJ incompetence and the control groups were analysed by the Mann-Whitney rank sum test [30] or as two samples of disparate size were being compared. Sensitivity, specificity, positive and negative predictive values were calculated for the SPECT/CT studies across the group with PGP/SIJ incompetence and the control group. The Student's $t$ test [30] was utilised for analysis of the significance of any difference between counts in the posterior soft-tissues in the group with PGP/SIJ incompetence. The Mann-Whitney rank sum test was used to analyse the comparison of counts between patients with PGP/SIJ incompetence and the groups with no back pain or non-specific low back pain [30]. Receiver operating characteristic curves were analysed for the SPECT/CT diagnostic criteria for PGP/SIJ incompetence [31]. The kappa score [32] was calculated for inter/intra-observer variability in reporting of the SPECT/CT studies. These studies were blindly reported by two senior nuclear medicine physicians with over 20 years experience. In this system of analysis, 0 represents no significant agreement and 1.0 represents perfect agreement.

\section{Results}

Population

The age demographics of the patients and the control group were assessed by the Mann-Whitney rank sum test due to the disparity in sample size. This yielded a Mann-Whitney $\mathrm{U}$ Statistic $=2,765.00$, indicating a non-significant difference between the two populations $(p=0.54)$. The index group of 100 patients had a history of trauma to the buttock or lower back in $52 \%$ of cases, either due to falls, complex work related trauma with rotational and compression injury or motor vehicle accidents. The remainder gave a similar history of pain in late pregnancy or in the post-partum period. Over $70 \%$ of cases gave a history of symptoms in excess of 2 years, with an average of 2.4 years. Over $75 \%$ of the cohort had reportedly normal MRI studies. This result was not formally assessed as part of the study as MRI has been shown to be of no utility in the condition. Patients with non-specific low back pain had no significant history of trauma and magnetic resonance imaging reported as normal in 45 cases, plain films and CT being reported as normal in five.

\section{Clinical testing}

The Stork test (stance phase), ASLR, posterior pelvic pain provocation test $(\mathrm{P} 4)$ and Gaenslen tests were positive in $100 \%$ of cases in the study cohort. Tests with a $90 \%$ positive rate were the palpation of the long dorsal sacroiliac ligament and modified Trendelenburg test. The study group fulfilled the criteria for PGP/SIJ incompetence with all patients returning a positive test of three or more of the four evidence-based criteria [4]. None of the control groups had a positive test for PGP/SIJ incompetence.

Planar bone scintigraphy

The blood pool images showed hyperaemia of the SIJ in $8 \%$ of patients with SIJ incompetence. The delayed planar study demonstrated increased uptake in the upper SIJ in $18 \%$ of these patients. In the control group, $12 \%$ of patients showed increased uptake in the inferior aspect of the SIJ and only two with sacroiliitis showed abnormalities in blood flow.

\section{SPECT/CT}

Scan criteria of increased uptake in the SIJ, increased posterior soft-tissue uptake and increased uptake at the site of sacroiliac ligament insertions on the ilium together with SIJ sclerosis on CT were found in 95 patients (Figs. 1, 2). By site, $53 \%$ were on the left, $36 \%$ on the right and $11 \%$ 

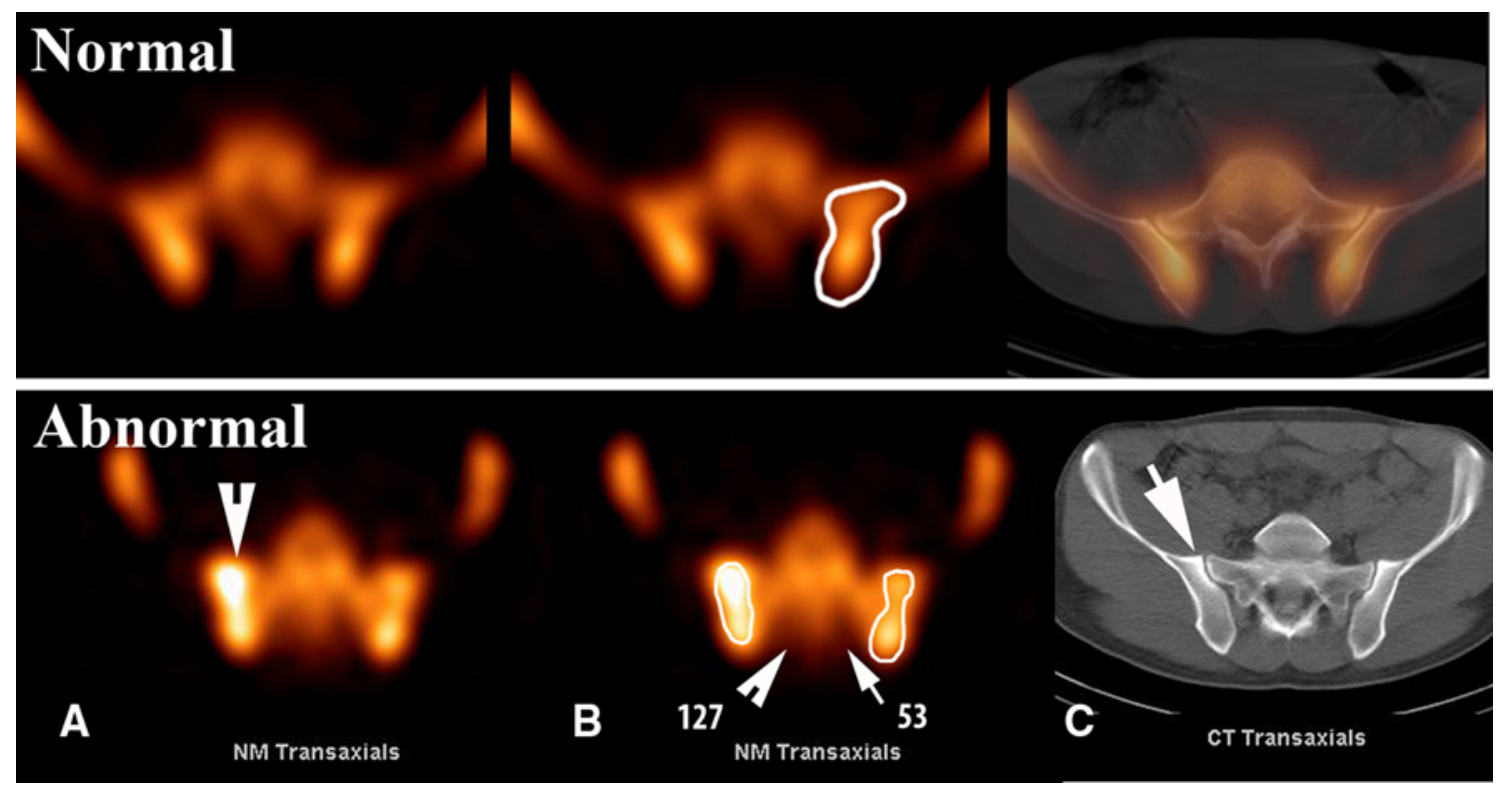

Fig. 1 Principal SPECT/CT manifestations of SIJ incompetence. Upper panel Pattern of uptake in normal SIJ. Note the "dumbbell" configuration of uptake in the SIJ. Lower panel Abnormal study. Intense uptake is evident in the right upper $(A)$ SIJ (arrowhead) with loss of the "dumbbell" configuration $(B)$ compared to the left side and

were bilateral. In the incorrect group, one patient was called on the contralateral asymptomatic side, two called bilateral and two normal. One case in the control group was called abnormal on the basis of uptake in the upper SIJ, without a significant difference in the posterior soft-tissue region of interest analysis. This was called normal in the second reading, but registered as a false positive case. Sensitivity was therefore $95 \%$ and specificity $99 \%$. Positive predictive value was $99 \%$ and negative predictive value $94 \%$.

Data for the analysis of regions of interest in the softtissues posterior to the SIJs passed the test of normality $(0.090)$ and equal variance $(0.209)$. The average counts on the abnormal side were 170 (SD: 92) and on the normal side 113 (SD: 68). Analysis of the counts on the abnormal versus the normal side by the $t$ test yielded a mean difference in counts of 57 (95\% CI: 12-91 counts) which was significant $(p=0.011)$. The $t$ test score was 2.60. The Mann-Whitney rank sum test was used to compare scores in patients with PGP/SIJ incompetence with patients with either no back pain or non-specific low back pain. A significant difference was also found between the abnormal side in patients with PGP/SIJ incompetence and patients with either no back pain or non-specific low back pain $(p=0.009)$. There was no significant difference between the normal side in patients with PGP/SIJ incompetence and patients with either no back pain or non-specific low back pain $(p=0.50)$. The power of the study was 1.0 . the normal study. $B$ Uptake extends into the posterior soft-tissues and ligamentous attachments on the ilium on the right side (arrowhead in $B$ ) with a higher photon count (127) compared to the uninvolved left side (53). $C$ There is bilateral SIJ sclerosis at approximately the S1/S2 segmental level, being more marked on the right (arrowhead)

Receiver operating characteristic curves were analysed for the SPECT/CT diagnostic criteria for SIJ incompetence and results are shown in Table 1 . While sensitive, the finding of SIJ joint uptake or sclerosis lacked specificity as these finding were also frequently found in patients with no back pain $(30 \%)$ or non-specific low back pain $(40 \%)$. The area under the curve (AUC) for this combination yielded a value of 0.41 . The best diagnostic criterion for SIJ incompetence was either soft-tissue uptake posterior to the joint $(\mathrm{AUC}=0.99)$ or uptake at the sacroiliac ligament insertions $(\mathrm{AUC}=0.99)$. The combination of these two criteria yielded an AUC of 1.0.

Additional findings on SPECT/CT included 31 patients with increased uptake at the site of adductor tendon insertion around the pubic symphysis (Fig. 3). Eight patients had bilateral uptake. Twenty-nine patients had increased uptake at the hamstring tendon insertion on the ischium (Fig. 2). Degenerative disease of the hips was evident in 19 patients and was graded as mild or moderate. The scintigraphic scan pattern of acetabular labral tears [33] and hip impingement (Fig. 2) [4] was evident in six and four patients, respectively, and was clinically not suspected. Gluteus medius tendon insertion enthesopathy was evident in two patients.

Patients with non-specific low back pain had evidence of lumbar zygapophyseal uptake at one or more levels in 40 of the 50 cases. These were graded as being of mild-moderate severity on SPECT/CT. Two patients had unilateral sacroiliitis 

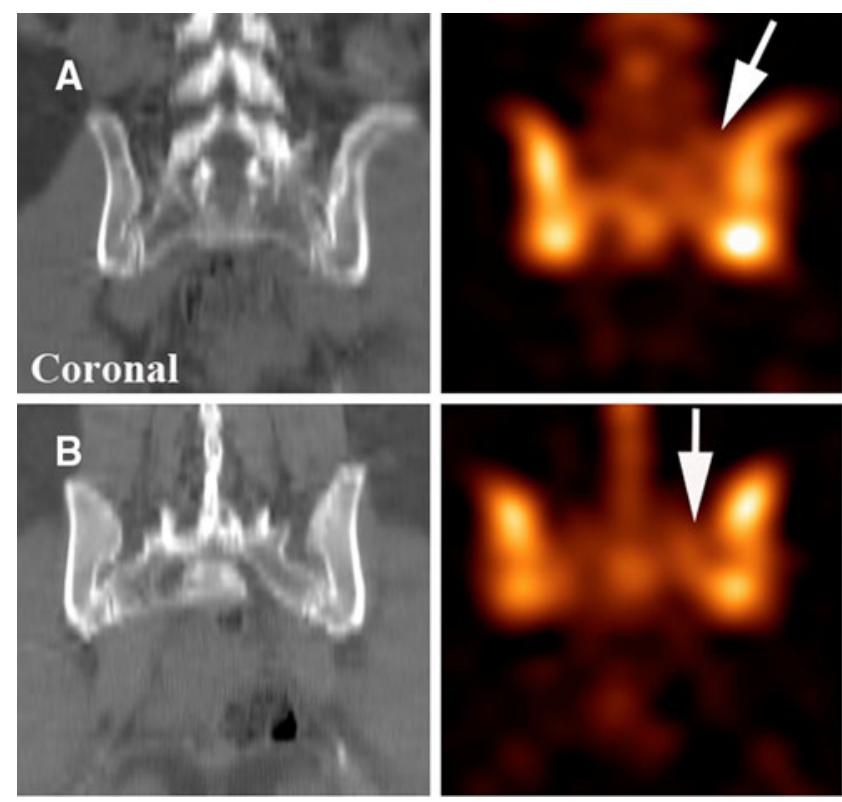

Fig. 2 Panel $A$ and $B$ demonstrate the pattern of soft-tissue uptake posterior to the left SIJ which is best appreciated in the coronal and transaxial images (arrows). This corresponds to the posterior ligament

Table 1 Performance of combined SPECT/CT findings

\begin{tabular}{llll}
\hline Finding & Sensitivity & Specificity & $\begin{array}{l}\text { Area under } \\
\text { curve (ROC) }\end{array}$ \\
\hline Soft-tissue uptake & 0.93 & 1.0 & 0.99 \\
Ligament enthesis uptake & 0.93 & 0.99 & 0.99 \\
SIJ uptake & 0.93 & 0.60 & 0.41 \\
SIJ sclerosis on CT & 0.75 & 0.60 & $\begin{array}{c}\text { (combined } \\
\text { finding) }\end{array}$ \\
\hline
\end{tabular}

by scintigraphic criteria. None had a pattern of uptake seen in the patients with PGP.

Inter/intra-observer variability for reporting the SPECT/ CT studies was good with kappa scores of 0.85 .

\section{Discussion}

The present study has identified a specific pattern of metabolic disturbances in and around the SIJs in patients with PGP/SIJ incompetence. These findings provide specific imaging evidence of significant correlative pathology as a platform of support for clinical testing as the current reference standard by which the diagnosis is established. Just as importantly, the imaging criteria are specific enough to separate PGP/SIJ incompetence from non-specific low back pain. The SPECT/CT findings reveal a unique diagnostic pattern which is loss of the normal "dumbbell" shape of scintigraphic uptake in the upper aspect of the SIJ
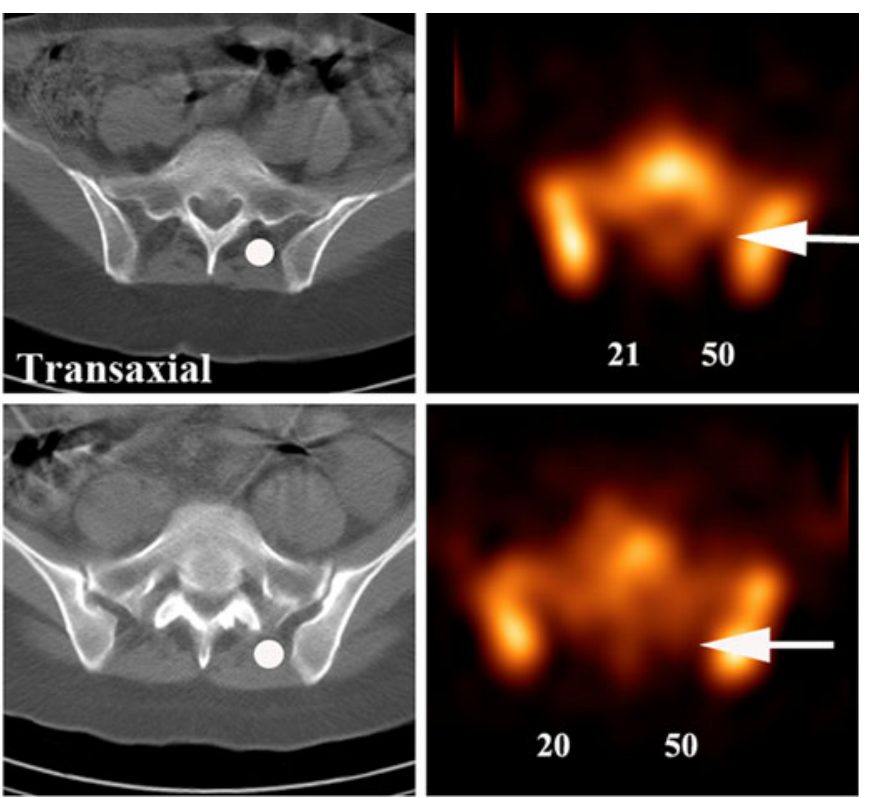

complex which in the CT images is clearly sited posterior to the joints. The counts are shown for left and right sides with the rounded white circles indicating the position from which counts were obtained

and the posterior soft-tissues (Figs. 1, 2). Increased softtissue uptake appears to be in the damaged posterior sacroiliac ligaments (Figs. 1,2). The average counts on the affected side were $50 \%$ higher than on the unaffected side. This is a significant finding with a power of 1.0, being highly unlikely to be a false positive difference. Furthermore, this is quite different to the patterns of uptake seen with degenerative disease, fractures, osteitis condensans ilii, metastatic disease and sacroiliitis [34]. Other findings such as adductor and hamstring uptake are less specific and likely to reflect the altered dynamics around the pelvis (Fig. 3), with joint sclerosis and uptake due to abnormal motion (counter-nutation, see Fig. 4c).

Patterns seen with degenerative disease include uptake in the inferior aspect of the SIJ, often with osteophyte formation. This may also be seen in the superior margin of the joint. Fractures usually extend vertically through the sacrum, parallel to the SIJ, with or without transverse extension across the sacrum ("H" or Honda sign). Osteitis condensans ilii is mild to moderate uptake on the iliac side of the SIJ with marked sclerosis that is usually symmetrical and variable in extent. Metastatic disease is focal, usually occurs in marrow containing bone in the pelvis and is away from the joint lines. It may be intense or "cold" depending on the grade of malignancy. Sacroiliitis usually occurs in the lower third of the SIJ and is associated with sclerosis, erosions and occasional joint ankylosis.

The scintigraphic changes when coupled with the pathophysiology of the condition allows a more meaningful appreciation of the anatomy and function of the SIJ 

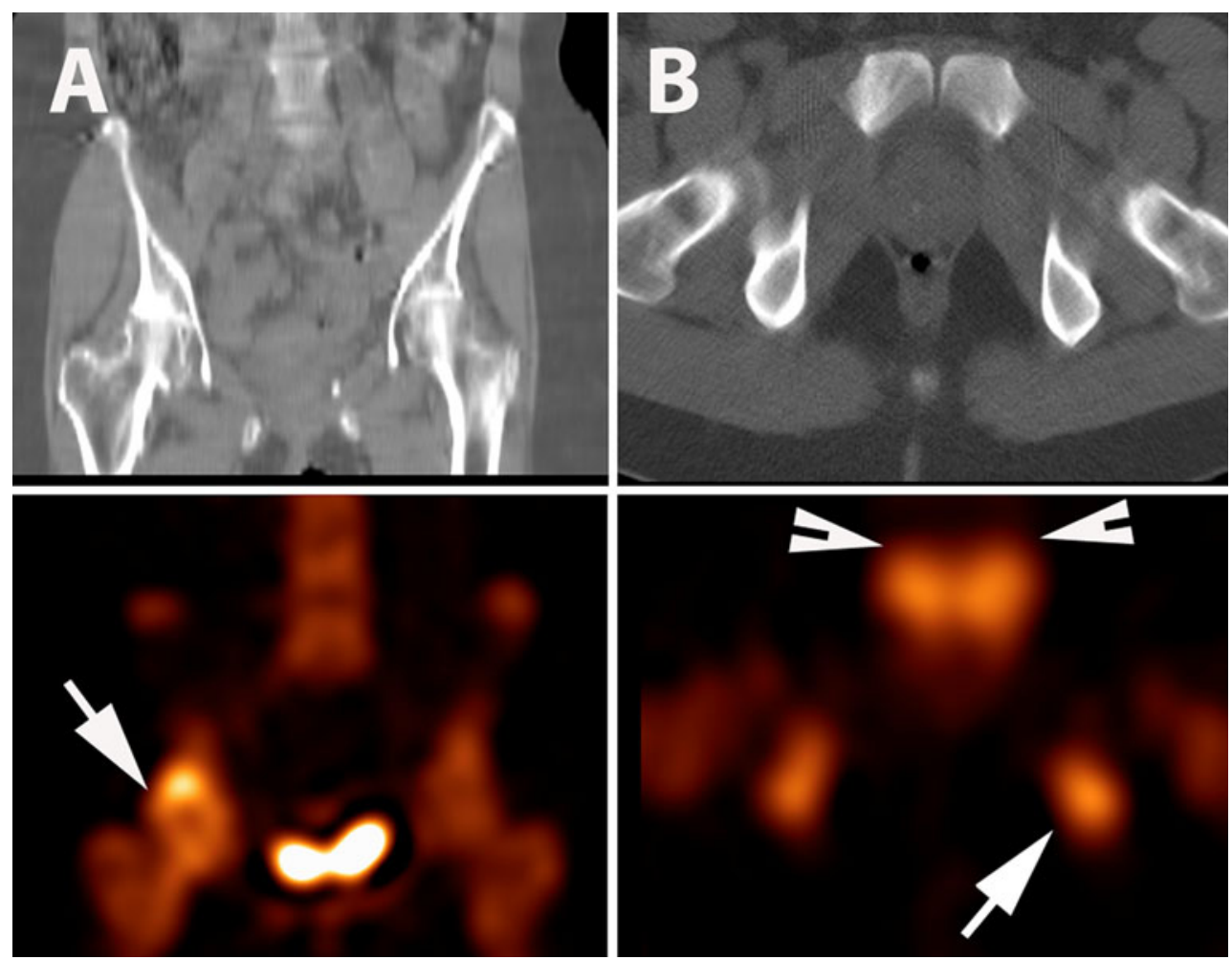

Fig. 3 Ancillary SPECT/CT findings of SIJ incompetence. a Right hip impingement with increased uptake and a "bump" on the lateral aspect of the femoral head (arrow). b Adductor (arrowheads) and hamstring (arrow) enthesopathy

Fig. 4 Illustrations of a form and force closure of the SIJ. b Nutation of the sacrum that allows the sacrum to fit into the pelvic ring in a stable configuration. c Counternutation occurs when the posterior ligamentous integrity is lost and the upper sacrum moves posteriorly

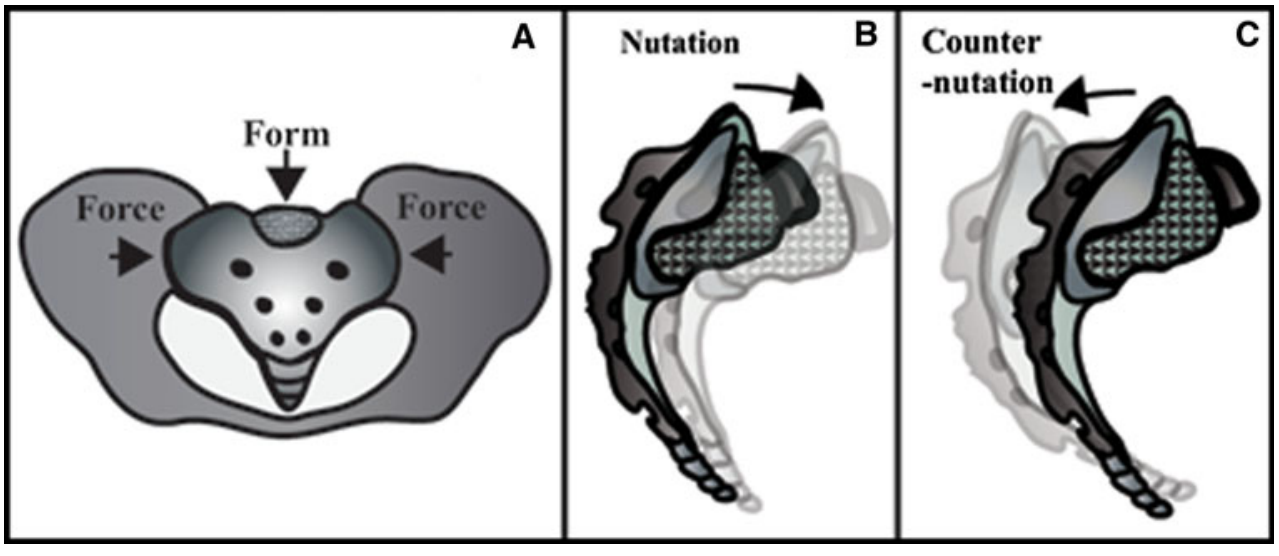

complex [35]. In functional terms, the SIJ is the linkage between the trunk and the lower limbs. The normal force distribution on the sacrum results in a net anterior moment on the sacrum [nutation (Fig. 4b)]. A complex interlacing arrangement of the dorsal sacroiliac, interosseous sacroiliac and sacrotuberous ligaments are critical in the posterior stabilisation of the joint [35]. The combination of matching articular surfaces and the stabilising influence of the ligaments provide what has been termed form closure of the joint (Fig. 3a) [36, 37]. Additionally, the combined action of a number of abdominal and pelvic muscles provides what has been termed force closure of the joint by a compressive mechanism on the pelvic ring (Fig. 4a) [36, 37].

Traumatic disruption of the posterior ligamentous complex or laxity induced by the hormonal changes of the peri-partum period may lead to a failure of form closure of the joint $[36,37]$. Abnormal sequencing of pelvic muscle contractility leads to failure of force closure of the joint [25]. Both mechanisms lead to abnormal posterior motion of the sacrum [counter-nutation (Fig. 4c)] and painful traction on the ligaments [38] as there is a failure of load transmission across the joint $[36,37]$. The source of pain 
arises from both the joint and the posterior ligamentous structures, not simply the joint as previously thought [38]. Hungerford et al. [25] have shown significant mechanically disadvantageous alterations in the patterns of recruitment of the lumbopelvic muscles by electromyographic studies in patients with pelvic pain. Cusi et al. [24] have shown significant improvements in the pain scores of patients with pelvic pain due to failure of load transfer across the SIJ treated with prolotherapy of the dorsal interosseous ligaments.

An equally significant finding of this study which has not previously been reported is the role of trauma. This is an important issue as the clinical diagnosis has been almost exclusively applied to a population of pregnant or postpartum women with the hormonal effect on the SIJ ligaments being purported as the likely aetiology. Well over half the population (52\%) with an established clinical diagnosis of SIJ incompetence in our series was due to trauma. The trauma fell into two prevalent patterns; either direct trauma by falls on to the buttocks or indirect trauma as part of motor vehicle accidents, where stationary cars were hit in the rear end and the patients had one leg anchored, leading to a twisting pelvic injury. The mechanism of injury is clearly different, yet the history, clinical and imaging findings are identical, suggesting that the final pathological pathway may well be identical. The term SIJ incompetence (failure of closure) has been derived from the nomenclature of cardiology, where a failure of cardiac valve closure is termed incompetence. A critical observation that adds weight to the assertion that the two conditions share a common pathophysiology is the response to specific therapy, with both aetiologies demonstrating a similar rate of response [24]. Identification of SIJ incompetence as a cause of pain is clinically important as it allows a specific therapeutic approach by either specialised physiotherapy [4, 39, 40], which is appropriate for some $80 \%$ of cases where muscle injury is the predominant issue or more controversially, prolotherapy for cases where ligamentous injury is the major contributory factor [24]. Rarely, more invasive measures such as fusion of the SIJ have also resulted in significant improvements in pain scores [41].

Acceptability of the condition in the wider medical community has been lacking for a number of reasons. The SIJ is relatively inaccessible to examination and a battery of often complex physical examinations are required that involve significant training and experience to master. It is only in the last few years that firm criteria for the physical tests have been established, validated and accepted [4]. In expert hands, these tests have been shown to have high inter-observer concordance with kappa values of between 0.81 and 1.00 [42]. In more generalised usage, the tests perform with less efficacy $[16,22]$. The absence of an adequate imaging test has added to this difficulty, as ultrasound [15] and scintigraphy [14] have been unable to provide clear and easily reportable criteria with good sensitivity and specificity. Furthermore, $75 \%$ of the patients with the clinically established diagnosis of PGP in the current study had normal MRI studies. Many of these patients expressed the view that they had been thought to be malingering or suffering from psychiatric disease, although this was not formally assessed in the current study.

Why is scintigraphy with SPECT/CT more efficacious than previously reported for SIJ incompetence [14] and why is it more suitable in this circumstance than MRI? The great advance in scintigraphy is the coupling of high contrast resolution cross-sectional images with the high spatial resolution of CT. Precise localisation of soft-tissue uptake can now be made more conspicuous with well registered anatomical and multi-planar functional images where both modalities are sequentially implemented without moving the patient. Reconstruction artefacts from radioactive urine in the bladder are less of an issue with statistical reconstruction (OSEM) compared with filtered back projection. Image clarity has been significantly improved with scatter correction and resolution recovery techniques that are standard on most devices. The fundamental pathology of SIJ incompetence is repetitive ligament micro-trauma leads to calcium deposition [43] and uptake of the scintigraphic agents in the ligament [44]. More advanced cases of the injury demonstrate ligament calcification in the CT study in several patients in the current study. The chronicity and absence of significant oedema and therefore a proton signal would reduce the utility of MRI. There is also repetitive injury at the ligament enthesis which is reflected in the scintigraphic study. In the long term, the repetitive abnormal motion (counter-nutation) of the SIJ results in a sclerotic reaction with increased uptake. The ancillary findings of adductor and hamstring enthesopathy and hip impingement are most likely due to the resultant alterations in muscle recruitment patterns [25]. The scintigraphic uptake mechanisms and the injury patterns are stereotypic and have been reported in similar soft-tissue injuries for over half a century [44].

\section{Conclusion}

Sacroiliac joint incompetence is a relatively common condition that may account for over $20 \%$ of low back pain, especially in patients in the peri-partum period or after pelvic trauma. Many of these patients have previously been classified as either non-specific low back pain or worse, as malingerers or manifestations of psychiatric disease. The clinical diagnosis requires meticulous attention to detail 
and expertise in physical examination that may be problematic in general usage. The majority of patients in this study had reportedly normal MRI studies, adding to the difficulty in identification by the standard medical paradigms. This study identifies associated metabolic disturbances which support the clinical findings. These are subtle changes that are often not evident in planar imaging and require meticulous attention to detail in interpretation of the SPECT/CT image sets.

Conflict of interest None.

\section{References}

1. Goldthwaite JE, Osgood RB (1905) A consideration of the pelvic articulation from an anatomical, pathological, and clinical standpoint. Boston Med Surg J 152:593-601

2. Mixter WJ, Barr JS (1934) Rupture of the intervertebral disc with involvement of the spinal canal. N Engl J Med 11:210-215

3. Dreyfuss P, Michalsen M, Pauza K (1996) The value of medical history and physical examination in diagnosin SIJ pain. Spine 21:2594-2602

4. Vleeming A, Albert H, Östgaard HC, Sturesson B, Stuge B (2008) European guidelines for the diagnosis and treatment of pelvic girdle pain. Eur Spine J 17(6):794-819

5. Albert H, Godskesen M, Westergaard J (2002) Incidence of four syndromes of pregnancy-related pelvic joint pain. Spine 27: 2831-2834

6. Larsen EC, Wilken-Jensen C, Hansen A, Jensen DV, Johansen S, Minck H, Wormsley M, Davidsen M, Hansen TM (1999) Symptom-giving pelvic girdle relaxation in pregnancy. I: prevalence and risk factors. Acta Obstetricia Gynecolog Scand 78: $105-110$

7. Ostgaard HC, Andersson GBJ, Karlsson K (1991) Prevalence of back pain in pregnancy. Spine 16:549-552

8. Maigne JY, Aivaliklis A, Pfefer F (1996) Results of sacroiliac joint double block and alue of sacroiliac pain provocation tests in 54 patients with low back pain. Spine 21:1889-1892

9. Schwarzer AC, Aprill CD, Bogduk N (1995) The sacroiliac joint in chronic low back pain. Spine 20:31-37

10. Dreyfuss P, Dreyer SJ, Cole A, Mayo K (2004) Sacroiliac joint pain. J Am Acad Orthop Surg 12:255-265

11. Weksler N, Velan GJ, Semionov M, Gurevitch B, Klein M, Rozentsveig V, Rudich T (2007) The role of sacroiliac joint dysfunction in the genesis of low back pain: the obvious is not always right. Arch Orthop Trauma Surg 127:885-888

12. Gupta AD (2009) Sacroiliac joint pathologies in low back pain. J Back Musculoskelet Rehabil 22:91-97

13. Margules KR, Gall EP (1997) Sciatica-like pain arising in the sacroiliac joint. J Clin Rheumatol 3:9-15

14. Slipman CW, Sterenfeld EB, Chou LH, Herzog R, Vresilovic E (1996) The value of radionuclide imaging in the diagnosis of sacroiliac joint syndrome. Spine 21:2251-2254

15. Saenz-Navarro I, Moller I, Iagnocco A, Naredo E (2011) Ultrasound assessment of the sacroiliac joint. J Clin Ultrasound 39:93-94

16. Cook C, Massa L, Harm-Ermandes I, Segneri R, Adcock J, Kennedy C, Figuers C (2007) Interrater reliability and diagnostic accuracy of pelvic girdle pain. J Manip Physiol Ther 30:252-258

17. Hodge JC, Bessette B (1999) The incidence of sacroiliac joint disease in patients with low-back pain. Can Assoc Radiol J 50:321-323
18. Kokmeyer D, van der Wurff P, Aufdemkampe G, Fickenscher TCM (2002) The reliability of multitest regimens with sacroiliac pain provocation tests. J Manip Physiol Ther 25:42-48

19. Laslett M, Aprill CN, McDonald B, Young SB (2005) Diagnosis of sacroiliac joint pain: validity of individual provocation tests and composites of tests. Man Ther 10:207-218

20. Mens JMA, Vleeming A, Snijders CJ, Koes BW, Stam HJ (2002) Validity of the active straight leg raise test for measuring disease severity in patients with posterior pelvic pain after pregnancy. Spine 27(2):196-200

21. Ostgaard HC, Zetherstrom G, Roos-Hansson E (1994) The posterior pelvic pain provocation test in pregnant women. Eur Spine J 3:258-260

22. Robinson HS, Brox JI, Robinson R, Bjelland E, Solem S, Telje T (2007) The reliability of selected motion - and pain provocation tests for the sacroiliac joint. Man Ther 12:72-79

23. van der Wurff P, Hagmeijera R, Meyne W (2000) Clinical tests of the sacroiliac joint. A systematic methodological review. Part 1: reliability. Man Ther 5:30-36

24. Cusi M, Saunders J, Hungerford B, Wisbey-Roth T, Lucas P, Wilson $P$ (2010) The use of prolotherapy in the sacro-iliac joint. Br J Sport Med 44(2):100104

25. Hungerford B, Gilleard W, Hodges P (2003) Evidence of altered lumbopelvic muscle recruitment in the presence of sacroiliac joint pain. Spine 28:1593-2000

26. Levangie PK (1999) Four clinical tests of sacroiliac joint dysfunction: the association of test results with innominate torsion among patients with and without low back pain. Phys Ther 79: 1043-1057

27. Mens JMA, Vleeming A, Snijders CJ, Koes BW, Stam HJ (2001) Reliability and validity of the active straight leg raise test in posterior pelvic pain since pregnancy. Spine 26:1167-1171

28. Schneider M, Erhard R, Brach J, Tellin W, Imbarlina F, Delitto A (2008) Spinal palpation for lumbar segmental mobility and pain provocation: an interexaminer reliability study. J Manip Physiol Ther 31:465-473

29. Hungerford B, Gilleard W, Moran M, Emmerson C (2007) Evaluation of the ability of physical therapists to palpate intrapelvic motion with the stork test on the support side. J Phys Ther 87:879-887

30. Fay M, Proschan M (2010) Wilcoxon-Mann-Whitney or $t$ test? On assumptions for hypothesis tests and multiple interpretations of decision rules. Stat Surv 4:1-39

31. Hanley J, McNeil B (1983) A method of comparing the areas under receiver operating characteristic curves derived from the same cases. Radiology 148:839-843

32. Cohen J (1968) Weighted kappa: nominal scale agreement with provision for scaled disagreement or partial credit. Psychol Bull 70:213-220

33. Bruce W, Van Der Wall H, Storey G, Loneragan R, Pitsis G, Kannangara S (2004) Bone scintigraphy in acetabular labral tears. Clin Nucl Med 29:465-468

34. Song IH, Carrasco-Fernandez J, Rudwaleit M, Sieper J (2008) The diagnostic value of scintigraphy in assessing sacroiliitis in ankylosing spondylitis: a systematic literature research. Ann Rheum Dis 67:1535-1540

35. Alderink GJ (1991) The sacroiliac joint: review of anatomy, mechanics, and function. J Orthop Sports Phys Ther 13:71-84

36. Lee D, Lee LJ (2010) The pelvic girdle: an integration of clinical expertise and research. Churchill Livingstone Elsevier, Edinburgh

37. Vleeming A, Stoeckart R, Volkers ACW, Snijders CJ (1990) Relation between form and function in the sacroiliac joint. Part I: clinical anatomical aspects. Spine 15:130-132

38. Murakami E, Tanaka Y, Aizawa T, Ishizuka M, Kokubun S (2007) Effect of periarticular and intraarticular lidocaine 
injections for sacroiliac joint pain: prospective comparative study. J Orthop Sci 12:274-280

39. Stuge B, Holm I, Vollestad N (2006) To treat or not to treat post partum pelvic girdle pain with stabilizing exercises? Man Ther 11:337-343

40. Stuge B, Morkved S, Dahl HH, Vollestad N (2006) Abdominal and pelvic floor muscle function in women with and without long lasting pelvic girdle pain. Man Ther 11:287-296

41. Buchowski J, Kebaish K, Sinkov V, Cohen D, Sieber A, Kostuik J (2005) Functional and radiographic outcome of sacroiliac arthrodesis for the disorders of the sacroiliac joint. Spine J 5:520-528
42. Albert H, Godskesen M, Westergaard J (2000) Evaluation of clinical tests used in classification procedures in pregnancyrelated pelvic joint pain. Eur Spine J 9(2):161-166

43. Hildebrand KA, Gallant-Behm CL, Kydd AS, Hart DA (2005) The basics of soft tissue healing and general factors that influence such healing. Sports Med Arthrosc Rev 13(3):136-144

44. Storey G, Murray I (2007) Nuclear Medicine in disorders of bones and joints. In: Ell P, Gambhir S (eds) Nuclear medicine in clinical diagnosis and treatment. Churchill Livingstone, Edinburgh 